\title{
Assessment of quay and yard transshipment operations under proximity limitations in multi-terminal container ports
}

\author{
Leonard Heilig \\ Univerity of Hamburg \\ leonard.heilig@uni-hamburg.de
}

\author{
Eduardo Lalla-Ruiz \\ University of Twente \\ e.a.lalla@utwente.nl
}

\author{
Christoph Bode \\ University of Hamburg \\ cbode.n7000@gmail.com
}

\author{
Stefan Voß \\ University of Hamburg \\ stefan.voss@uni-hamburg.de
}

\begin{abstract}
The assignment of storage locations and space has a considerable impact on the performance of container terminals. This holds especially true in multi-terminal transshipment ports where the planning of inbound and outbound container flows needs to consider space limitations and travel distances for reallocations, causing both intra- and inter-terminal transports. Thus, in this work, we study the impact of closeness limitations on quay and yard areas when conducting transshipment operations at multi-terminal transshipment ports. In doing so, a mathematical formulation and several scenarios covering different distance policies for limiting the allocation of containers before vessel loading or unloading operations are assessed. At a tactical level, this paper provides insights on assignment decisions while assessing distance-based policies that can be incorporated in practice.
\end{abstract}

\section{Introduction}

Maritime transport and the international shipping industry are the backbone of globalized supply chains and therefore have a significant role in international trade. Aside from being forced to have investments to accommodate ever-increasing vessels, e.g., by deepening channels and constructing new berthing facilities, operational practices need to be constantly improved. For that reason, it is crucial that the handling of containers is efficiently managed during vessel loading and unloading operations as well as during their stay in the storage yard as those factors directly influence the costs of terminal operations. Firstly, the arriving vessels, which will moor at the quay, need to be allocated to berthing positions. The planning of this operation leads to the well-known berth allocation problem (BAP), whose goal is to determine an optimal berthing position and time for a vessel under a given objective. Secondly, in order to speed-up the (un-)loading process, transshipment containers often need to be reallocated to a yard position close to the vessel's berthing position. Optimizing the container allocation within the storage yard is referred to as the container stacking problem or yard allocation problem (YAP). Given that many ports have several terminals, this reallocation of containers may lead to inter-terminal transports that largely contribute to the operational costs [1], service time [2, 3, 4], and environmental impact $[5,6]$. In particular, the travel distances between quayside and yardside as well as between yards have an essential impact on the overall costs and performance, especially when inbound and outbound flows need to be coordinated in transshipment terminals, and thus need to be minimized (see, e.g., [7]). The optimization of multiple terminals has received fairly little attention in the academic literature so far, even though it is required to increase the port's overall performance [8] and addresses the fact that multiple terminals are managed by one operator in an increasing number of ports [9]. Individually, the BAP and storage space optimization have been studied extensively. Due to the interrelation between those two problems as described above, integrated approaches for simultaneously solving the BAP and YAP have been receiving more academic attention recently. However, it is important to treat these intertwined problems in an integrative fashion and assess the impact of distance limitations in order to provide decision support and policies in transshipment operations.

In this paper, we aim to enhance the understanding and use of the impact of closeness limitations and costs of operations on the terminal and yard assignment problem (TYAP). In doing so, we use the TYAP optimization model presented in [1] which aims to minimize the total inter-terminal and intra-terminal handling costs generated by transshipment flows. We use this integer programming model while considering (i) container groups' travel distances rather than costs and (ii) closeness restrictions for loading or unloading container groups between quay and yard areas instead of travel cost limitations. The closeness among blocks 
and terminals can be quantitative (e.g., distance) or qualitative (e.g., set by managers). Subsequently, we conducted a sensitivity analysis to determine the role of proximity limitations within the assignment decisions based on the different components of the objective function. This analysis provides insights with respect to yard allocation strategies where the proximity between quay and yard is seen as a relevant factor, in particular in transshipment terminals, as it accounts for the availability of resources performance of the terminal. The computational results are discussed in terms of the objective values, computational time, and practical implications.

The paper is structured as follows. Section 2 gives an overview of related works tackling the integration of berth and yard assignment problems. In Section 3, we introduce the considered problem by means of a mathematical formulation. The computational results of the sensitivity analysis are presented and discussed in Section 4. The managerial implications derived from the results are discussed in Section 5. Finally, concluding remarks and plans for further research are outlined in Section 6.

\section{Related works}

The BAP and YAP are two highly interrelated problems. For the BAP, berthing positions depend on the decisions made in the YAP, namely the storage location of the containers and resulting handling times. On the other hand, for most studies regarding the YAP, it is assumed that berthing positions and time windows are known a priori in order to plan the assignment of storage space to containers. Integrated approaches try to overcome this dilemma by considering both problems simultaneously which leads to higher computational complexity but overall better results. Even though the integration of the BAP and YAP is an important practical issue, only a few approaches are currently available in the literature. As presented in the following, the results of quantitative studies in this context underline the impact of this type of integration on the terminal's performance.

One of the first approaches for solving the YAP and the BAP in an integrated fashion has been proposed by Safaei et al. [10]. They formulate a two-level approach where the mathematical programming models are solved consecutively with the overall objective of minimizing the transport costs of import containers. They consider a dynamic rolling-horizon approach in which the next planning horizon begins with a new vessel's arrival. The first level handles the storage allocation of different container numbers and types (i.e., regular or refrigerated containers) to blocks in order to minimize their storage and retrieval times. The results serve as an input for the second level which assigns the vessels to berths with the goal of minimizing the travel distance between storage blocks and berthing locations. The results show that their model provides cost savings of up to $7 \%$ compared to real assignment plans, which might lead to a competitive advantage for terminal operators.

Salido et al. [11] develop several algorithms to solve the container stacking problem and the BAP independently. For the proposed stacking algorithms, the main objective is to minimize the number of reshuffles and reallocations. Other objectives, e.g., reducing the distance to the cargo sites as well as safety requirements, can also be taken into account. The goal of their heuristic for a dynamic BAP is to minimize the total weighed waiting time of vessels. The authors develop an iterative procedure that solves the BAP before the stacking problem and involves several iterations and feedback loops until a solution is found. They further discuss the trade-off between considered goals (i.e., number of reshuffles vs. total waiting time) and the use of weights in practice depending on port priorities.

Hendriks et al. [12] propose an integrated approach at a tactical level. Building on a previous study (see [9]), the authors assume that a robust terminal assignment and cyclic berth planning has been determined on the strategic level beforehand so that arrival and departure times of vessels are known a priori. They formulate a mixed-integer quadratic model to dynamically allocate different numbers and types of containers to storage blocks and vessels to the continuous quay with the objective of minimizing the overall prime movers' travel distance. Since this formulation has multiple local minima, the authors propose a relaxed model to provide a suitable starting point for their heuristic which alternates between solving the BAP and YAP until a stopping criterion is satisfied. The authors evaluate their approach with a data set from the Port of Antwerp, Belgium, and show that the optimization approach can considerably reduce the total travel distance.

Some of the integrated approaches consider container transshipment hubs. Zhen et al. [13] simultaneously design a yard template and a berth template, including the assignment of quay cranes, for cyclical calling vessels. Supporting the tactical decision making, those templates can be used for the pre-planning of recurring terminal operations. The yard template handles the reservation of sub-blocks for containers to be transshipped between vessels. The objective of their mathematical model is to minimize 
the weighed service costs, depending on vessels' deviation from the expected turnaround time intervals and the operation cost which depends on the length of transshipment flows. To solve the problem, the authors develop a heuristic that first solves the problems separately and then integrates their solutions through an iterative alternating procedure. Computational results show that the integration of the berth and yard template can reduce the transportation distance up to $27 \%$ compared to less sophisticated planning strategies.

While Lee et al. [14] assume that the spatial decision of the BAP serves as an input, they consider the temporal decision of determining optimal calling schedules of feeder vessels for optimizing the storage yard allocation problem. The goal of their proposed model is to minimize the length of transshipment flows and to balance the workload between shifts. An evenly distributed workload is preferred because a large imbalance would lead to either times with excessive workload or idle times of the handling equipment. The results show that there is a significant improvement in terms of workload balance and travel distance of the containers when an adjustment of the feeder calling schedule is allowed. However, it must be noted that their approach cannot be classified as integrative.

Lee et al. [1] introduce the TYAP aiming to optimally allocate vessels to terminals and container groups to the storage yard on a tactical level in order to minimize the handling costs of transshipment flows. In their study, the authors perform a sensitivity analysis regarding the parameter $r_{k}$. This parameter limits the number of possible reallocation movements for container groups during their duration of stay within the storage yard. Moreover, a two-level heuristic is presented for solving the integrated problem in an efficient way.

A transshipment problem similar to [14] is studied in Lee and Jin [15]. They assume that for a given calling schedule of mother vessels ${ }^{1}$, the terminal operator can proactively design the calling schedule of feeder vessels. Thus, in their study, they integrate the tactical problem of designing a berth, yard and schedule template for feeder vessels. With given berthing positions and times for the mother vessels, the proposed model determines these variables for feeder vessels and assigns the transshipment container flows to yard sections. To handle the NP-hardness, the authors develop a memetic heuristic which can obtain close-to-optimal solutions for large instances efficiently. Jin et al. [16] reformulate the mixed-integer programming model as a set covering

\footnotetext{
${ }^{1}$ In ocean shipping networks, a mother vessel is a seagoing ship that only serves transshipment hubs in major ports. To connect smaller ports, cargo needs to be transshipped from the mother vessel to smaller feeder vessels.
}

model and develop a column generation approach that outperforms the memetic heuristic of [14] with respect to solution quality and especially computational efficiency.

Zeng et al. [17] integrate the berth allocation and storage space assignment on an operational level and include the concept of direct transshipments (i.e., containers from one vessel can be directly loaded onto another vessel without being stored). In their model, the (indirect) transshipment container volumes are assigned to different blocks in the yard, and the predetermined location of export containers is also taken into account for assigning vessels to berthing positions and times along the continuous quay. In their model, the authors only consider direct transshipments from feeder to mother vessels. Thus, the completion time of unloading operations for a mother vessel and the berthing times of feeder vessels need to be synchronized. The goal is to minimize the delay cost of vessels and the total transshipment costs by also considering the cost savings of yard cranes resulting from direct transshipment. The authors develop an alternating two-level heuristic based on a genetic algorithm, in which the first level determines the berth allocation and direct transshipment plan and the second level handles the storage space optimization. Computational results show that the cost can be reduced up to $22 \%$, compared to models that only consider the BAP.

\section{Problem description and mathematical model}

The TYAP occurs in ports where multiple container terminals are located close to each other and which are sometimes operated by the same terminal operator. An example is the Port of Hamburg, Germany, where three of four container terminals are managed by one terminal operator. In a multi-terminal system, operations are more complex than in a single terminal. Especially in ports and terminals with severe space limitations, like in Hamburg, unnecessary container moves and an inefficient assignment of storage capacities may aggravate problems, such as with regard to traffic congestion and environmental issues. Moreover, cost for storing containers, referred to as demurrage fees, might become due when exceeding the free time (usually about three days) of container storage in individual yards. Therefore, it is essential to efficiently plan the movement and storage of transshipment containers to satisfy vessel calls, also by taking into account the cases where containers arrive at one terminal and leave the port from another terminal causing inter-terminal transportation (see [4]). 


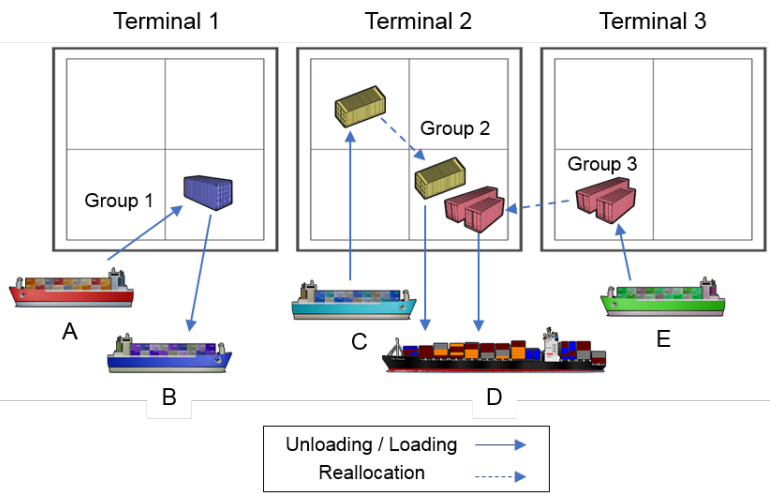

Figure 1. Example of a multi-terminal transshipment hub with 3 terminals and 5 container vessels

As described in the previous sections, the issue of allocating yard storage space and managing container flows is highly related to the allocation of the vessels. The containers need to be assigned to storage areas when they are discharged from an arrived vessel. Before the outgoing vessel arrives, they need to be moved to yards close to the berthing position of the departing vessel in order to speed up the loading process. In a transshipment hub with limited storage space, reallocations of containers might be necessary during their duration of stay. While reallocations between two yards inside a terminal lead to intra-terminal costs, reallocations between terminals cause inter-terminal costs. The TYAP, in this work, aims to minimize both intra- and inter-terminal distances while allocating the containers to areas in the storage yard. Figure 1 illustrates a small example with 3 terminals and 3 container groups that have to be exchanged between vessels to continue their corresponding route.

The TYAP can be modeled as a space-time network optimization problem. All necessary notations and decision variables are depicted in Tables 1 and 2, respectively. As depicted in Figure 2, the flow of a container group $k$ can be represented by a path from a source node $S_{k}$ to a sink node $T_{k}$, indicated by the solid arrows. A container group arrives with an inbound vessel in $t=a_{k}$ and departs with an outbound vessel in $t=b_{k}$. Inside the yard, it is possible to reallocate a container group from one yard area $i$ to another yard area $j$ using the decision variable $X$. The example shows that the container group can also remain in one yard area for several periods. While the dotted arrows between terminals and yards represent movements between the quayside and yard areas, those inside yards indicate the reallocations of container groups between successive time periods. Each arc defines a distance between the pair of linked nodes and
Table 1. Indices and parameters

\begin{tabular}{|c|c|}
\hline$i, j$ & index for terminals and yards \\
\hline$k$ & index for container groups \\
\hline$t$ & index for time periods \\
\hline$M$ & set of storage yards, $M=\{1,2, \ldots, \bar{m}\}$ \\
\hline$N$ & set of terminals, $N=\{1,2, \ldots, \bar{n}\}$ \\
\hline$T$ & set of time periods \\
\hline$K$ & set of container groups \\
\hline$a_{k}$ & arrival of group $k, a_{k} \in T$ \\
\hline$b_{k}$ & departure of group $k, b_{k} \in T$ \\
\hline $\begin{array}{l}q_{k} \\
v_{k}^{1} \\
v_{k}^{2}\end{array}$ & $\begin{array}{l}\text { storage space requirement of group } k \\
\text { inbound vessel of group } k \\
\text { outbound vessel of group } k\end{array}$ \\
\hline$r_{k}$ & $\begin{array}{l}\text { maximum allowed reallocations between } \\
\text { yards for group } k\end{array}$ \\
\hline$Q_{i}^{1}$ & storage capacity of yard $i, i \in M$ \\
\hline$Q_{i}^{2}$ & $\begin{array}{l}\text { processing capacity of terminal } i \text { in a time } \\
\text { period, } i \in N\end{array}$ \\
\hline$\alpha_{k l}$ & $\begin{array}{l}\text { set to } 1 \text { if group } k \text { and } l \text { have the same } \\
\text { inbound vessel (i.e., } o_{k}=o_{l} \text { ), and } 0 \\
\text { otherwise, } k, l \in K\end{array}$ \\
\hline$\beta_{k l}$ & $\begin{array}{l}\text { set to } 1 \text { if group } k \text { and } l \text { have the same } \\
\text { outbound vessel (i.e., } d_{k}=d_{l} \text { ), and } 0 \\
\text { otherwise, } k, l \in K\end{array}$ \\
\hline$\gamma_{k l}$ & $\begin{array}{l}\text { set to } 1 \text { if the inbound vessel of group } k \text { and } \\
\text { the outbound vessel of group } l \text { are the same } \\
\text { (i.e., } o_{k}=d_{l} \text { ), and } 0 \text { otherwise, } k, l \in K\end{array}$ \\
\hline$\delta$ & $\begin{array}{l}\text { maximum allowed closeness between quay } \\
\text { side and yard side }\end{array}$ \\
\hline$d_{i j}^{1}$ & $\begin{array}{l}\text { travel distance between yard } i \text { and yard } j \text {, } \\
i, j \in M\end{array}$ \\
\hline$d_{i j}^{2}$ & $\begin{array}{l}\text { travel distance between terminal } i \text { and yard } \\
j, i \in N, j \in M\end{array}$ \\
\hline$c_{i j}$ & $\begin{array}{l}\text { closeness between terminal } i \text { and yard } j, i \in \\
N, j \in M\end{array}$ \\
\hline
\end{tabular}

can be either an intra- or inter-terminal movement. The terminal operator can specify the maximum number of reallocations $r_{k}$ inside yards and, as done in our current problem variant, the maximum closeness $\delta$ between yard areas and quayside. While it has been shown in [1] that varying the parameter $r_{k}$ provides no significant improvements, but leads to higher computational times, in this work, we define a closeness index to analyze the impact of policies regarding closeness limitations on different components of the objective function and computational performance.

The mathematical model of the TYAP used in this work is mostly based on the one presented in [1]. Nevertheless, our mathematical model considers (i) travel distances in the objective function and (ii) closeness limitations between quay and yard for loading 
Table 2. Decision variables

\begin{tabular}{ll}
$X_{i j k}^{t}$ & set to 1 if group $k$ is located at yard $i$ at time period $t$ and located at yard $j$ at time period $t+1$, and \\
& 0 otherwise, $i, j \in M, k \in K, t \in T$ \\
$U_{i j k}^{t}$ & set to 1 if group $k$ uses arc $i \rightarrow j$ at time period $t$ upon arrival, and 0 otherwise, $i \in N, j \in M, k \in$ \\
& $K, t \in T$ \\
$V_{i j k}^{t}$ & set to 1 if group $k$ uses arc $i \rightarrow j$ at time period $t$ upon departure, and 0 otherwise, $i \in M, j \in$ \\
& $N, k \in K, t \in T$ \\
$W_{i j k}^{1}$ & set to 1 if group $k$ is located at yard $i$ at time period $t$, and 0 otherwise, $i \in M, k \in K, t \in T$ \\
$W_{i j k}^{2}$ & set to 1 if group $k$ is processed (i.e., loaded or discharged) at terminal $i$ at time period $t$, and 0 \\
& otherwise, $i \in N, k \in K, t \in T$ \\
$Z_{i k}^{1}$ & set to 1 if group $k$ uses terminal $i$ upon arrival, and 0 otherwise, $i \in N, k \in K$ \\
$Z_{i k}^{2}$ & set to 1 if group $k$ uses terminal $i$ upon departure, and 0 otherwise, $i \in N, k \in K$ \\
\hline
\end{tabular}

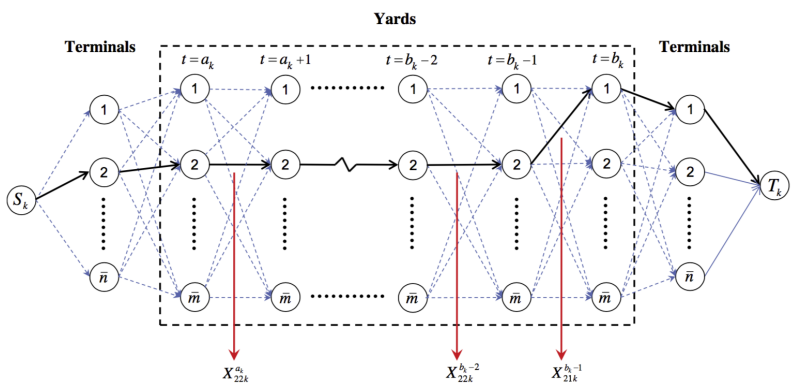

Figure 2. Time-space network of the TYAP for a container group [1]

and unloading operations. In doing so, for each terminal $i \in N$ and each storage yard $j \in M$, an integer value $c_{i j}$ is defined. By means of this variable, a value of $c_{i j}=1$ means that a yard block $j$ is at the closest position to the quayside of terminal $i$. Thus, higher values of $c_{i j}$ indicate more distant locations. Note that this closeness factor can be defined either in a quantitative way (e.g. according to distances or costs) or a qualitative way (e.g. depending on the yard distribution, managerial strategies, etc.). Thus, in this work, for each terminal and yard block, we consider that for a given position there is a subset of predefined terminals and yard locations defined as very near (i.e. $c_{i j}=1$ ) as well as we may have other farther (i.e. $c_{i j}>1$ ). Based on this, our model differs from [1] in the objective function as we consider distances instead of costs and in the restriction for loading and unloading containers from the quay to the yard, and vice-versa, which is given by a closeness measure. Our extended formulation is stated as follows.

$$
\begin{array}{r}
\min \left\{\sum_{k \in K} \sum_{i \in M} \sum_{j \in M} \sum_{t \in T} d_{i j}^{1} q_{k} X_{i j k}^{t}+\right. \\
\left.\sum_{k \in K} \sum_{i \in N} \sum_{j \in M} \sum_{t \in T} d_{i j}^{2} q_{k}\left(U_{i j k}^{t}+V_{j i k}^{t}\right)\right\}
\end{array}
$$

subject to the following constraints:

$$
\begin{aligned}
& \sum_{i \in N} Z_{i k}^{1}=1 \forall k \in K \\
& \sum_{i \in N} Z_{i k}^{2}=1 \forall k \in K \\
& \sum_{i \in M} X_{j i k}^{t+1} \forall j \in M, k \in K \\
& a_{k} \leq t \leq b_{k}-2
\end{aligned}
$$$$
\sum_{i \in M} X_{i j k}^{t}=\sum_{i \in M} X_{j i k}^{t+1} \forall j \in M, k \in K,
$$

$$
Z_{i k}^{1}=\sum_{j \in M} U_{i j k}^{t} \forall i \in N, k \in K, t=a_{k}
$$

$$
\sum_{i \in N} U_{i j k}^{t}=\sum_{i \in M} X_{j i k}^{t} \forall j \in M, k \in K, t=a_{k}
$$

$$
\sum_{j \in N} V_{i j k}^{t}=\sum_{j \in M} X_{j i k}^{t-1} \forall i \in M, k \in K, t=b_{k}
$$

$$
\sum_{i \in N} \sum_{j \in M} c_{i j} U_{i j k}^{t} \leq \delta \forall k \in K, t=a_{k}
$$

$$
\sum_{i \in M} \sum_{j \in N} c_{j i} V_{i j k}^{t} \leq \delta \forall k \in K, t=b_{k}
$$

$$
W_{i k t}^{1}=\sum_{j \in M} X_{i j k}^{t} \forall i \in M, k \in K, a_{k} \leq t \leq b_{k}-1
$$




$$
\begin{gathered}
W_{i k t}^{1}=\sum_{j \in N} V_{i j k}^{t} \forall i \in M, k \in K, t=b_{k} \\
W_{i k t}^{2}=\sum_{j \in M}\left(U_{i j k}^{t}+V_{j i k}^{t}\right) \forall i \in N, k \in K, t \in T \\
\sum_{k \in K} q_{k} W_{i k t}^{1} \leq Q_{i}^{1} \forall i \in M, t \in T \\
\sum_{k \in K} q_{k} W_{i k t}^{2} \leq Q_{i}^{2} \forall i \in N, t \in T \\
\sum_{t \in T} \sum_{i \in M} \sum_{j \in M \mid j \neq i} X_{i j k}^{t} \leq r_{k} \forall k \in K \\
\alpha_{k l}\left(Z_{i k}^{1}-Z_{i l}^{1}\right)=0 \forall i \in N, k \in K, l \in K, k \neq l \\
\beta_{k l}\left(Z_{i k}^{2}-Z_{i l}^{2}\right)=0 \forall i \in N, k \in K, l \in K, k \neq l \\
\gamma_{k l}\left(Z_{i k}^{1}-Z_{i l}^{2}\right)=0 \forall i \in N, k \in K, l \in K, k \neq l \\
X_{i j k}^{t}, U_{i j k}^{t}, V_{i j k}^{t}, W_{i k t}^{1}, W_{i k t}^{2}, Z_{i k}^{1}, Z_{i k}^{2} \in\{0,1\}
\end{gathered}
$$

The objective function of the TYAP model (1) seeks to minimize the distance for reallocating container groups within the yard as well as for moving container groups between the quay and the yard areas. Constraints (2) and (3) ensure that each container group arrives and departs at exactly one terminal, respectively. Constraints (4) handle the flow conservation for container group reallocations between yard areas. That is, before a container group can be reallocated from a yard area $j$ to another yard area $i$, it needs to be allocated to yard area $j$ beforehand. The linking constraints (5) and (6) link decision variables $U, V$, and $Z$ regarding the assignment of a terminal and the assignment of an initial or final yard area, respectively. Constraints (7) and (8) ensure the flow conservation between the quayside and yard area and link the decision variables $U, V$, and $X$. Constraints (9) and (10) set a restriction on the maximum distance for moving container groups between quay and yard areas based on the closeness index $c_{i j}$. Constraints (11)-(13) define the decision variable $W$. While constraints (11) set that a container group has been stored in a yard area $i$ when reallocated to another yard area $j$, constraints (12) ensure that a container group has been stored in a yard area $i$ before leaving through terminal $j$. Constraints (13) make sure that the container group needs to be processed at the quayside (i.e., loaded or discharged) when it arrives or departs at a terminal $i$. The storage space and quayside processing restrictions are defined in constraints (14) and (15). Constraints (16) set a restriction regarding the number of reallocations inside and between yards. Terminal allocation constraints for groups of the same inbound/outbound vessels are considered by constraints (17)-(19). Finally, constraints (20) define the decision variable types.

\section{Sensitivity analysis and numerical results}

In this section, we present the results of a sensitivity analysis for the above described problem. For this purpose, the presented model has been implemented and used to conduct different experiments. Before explaining the computational results and lessons learned, this section briefly introduces the design of the experiments and the problem instances being used to evaluate different scenarios.

\subsection{Experimental design}

Based on the extended TYAP model, the sensitivity analysis comprehensively studies the influence of the proximity limitations on the optimization results and computational times. The parameter $\delta$ limits the closeness between the quay of the terminal and the storage yard in which the container groups must be stored upon arrival/departure. Upon the berthing of a vessel, its incoming and outgoing container groups can be directly stored at or retrieved from one of the yard areas within this proximity. Given the limited processing capacity of terminals, the storage capacity of yards, and the fact that incoming and outgoing vessels of a container group might be allocated to different terminals, container reallocations may become necessary in order to not exceed the maximum transport distance upon berthing.

In general, it is advisable to store container groups close to the berthing position of a vessel in order to speed up quayside handling operations. Nevertheless, the case of $\delta=\infty$ is considered in the following analysis even though this case might be impractical in reality, as it allows container groups to be stored at the farthest position possible upon unloading/loading operations. Additionally, $\delta$ is set to 1,2 , and 3 , which represents different assumptions regarding the maximum closeness distance from the berthing 
vessel. The rationale behind this is to provide implications for choosing the best managerial policy regarding proximity limitations concerning the loading and unloading positions in multi-terminal transshipment ports. The different settings of $\delta$ restrict the use of yard areas as follows:

- $\delta=1$ : Only the closest yard areas of the terminal $\left(c_{i j}=1\right)$.

- $\delta=2$ : All yard areas smaller or equal to a closeness of two $\left(c_{i j} \leq 2\right)$. In the example shown in Figure 1, this case is represented by Group 2 .

- $\delta=3$ : All yard areas having a value $c_{i j} \leq 3$. If we would consider the case that the movement to a neighboring terminal increments $c_{i j}$ by one, Group 3 can be an example of this case in Figure 1.

- $\delta=\infty$ : No distance limitation.

For the largest cases of $\delta$, i.e., $\delta=3$ and $\delta=\infty$, the container groups can be directly unloaded to or loaded from any yard within the same terminal, and additionally from the closest yard of the neighboring terminal. This means that inter-terminal transport movements are possible during the unloading or loading of a vessel, which is considered to be impracticable in reality due to the long distances between terminals, leading to prolonged handling times of the vessels. On the other hand, in the scenario of $\delta=2$, the groups must be stored within the same terminal as the scheduled vessel upon its arrival or departure. For $\delta=1$ the situation is the most restricted, as the groups must be stored in one of the two yards closest to the quayside of the terminal that the scheduled vessel is berthing at. Aside from the variation of $\delta$, the sensitivity analysis considers a separation of the objective function into both of its parts to gain insights on the impact of each objective on the overall result and computational performance:

- $O b j_{1}$ : Minimization of the travel distance resulting from container reallocations inside the yard.

- $\mathrm{Obj}_{2}$ : Minimization of the travel distance before or after vessel discharge or loading operations, respectively.

To conduct the sensitivity analysis, a set of 12 randomly generated test instances has been used. The number of groups ranges from 5 to 60 groups with some groups arriving/departing within the same vessels. With the assumption that a time period has the length of 8 hours (i.e., one working shift), the planning horizon is 6 to 9 time periods (i.e., two to three days) for the smaller instances and 21 time periods (i.e., one week) for the larger ones. We further use the port layout presented in [1] with three terminals having four storage yard areas each. Finally, the $c_{i j}$ values are generated according to the previous description of $\delta$.

\subsection{Computational results}

This section presents the computational results of the conducted numerical experiments. The model presented in Section 3 is implemented and solved in CPLEX 12.6.3, and all computations are conducted on a computer with an Intel i5 $3.5 \mathrm{GHz}$ and $16 \mathrm{~GB}$ of RAM. The executions were limited to use only one CPU.

Table 3. Computational results

\begin{tabular}{rrrrr}
\hline \multicolumn{5}{c}{$A: \min \left(O b j_{1}+O b j_{2}\right)$} \\
\hline$\delta$ & $O b j_{1}+O b j_{2}$ & $O b j_{1}$ & $O b j_{2}$ & Time (s) \\
\hline 1 & 20.14 & 4.22 & 15.92 & 33.35 \\
2 & 19.52 & 2.27 & 17.25 & 48.02 \\
3 & 18.84 & 0.74 & 18.10 & 36.34 \\
$\infty$ & 18.84 & 0.31 & 18.53 & 37.23 \\
\hline \multicolumn{5}{c}{$B: m i n\left(O b j_{1}\right)$} \\
\hline$\delta$ & $O b j_{1}+O b j_{2}$ & $O b j_{1}$ & $O b j_{2}$ & Time (s) \\
\hline 1 & 20.14 & 4.22 & 15.92 & 9.84 \\
2 & 24.52 & 0.75 & 23.77 & 91.73 \\
3 & 30.24 & 0.00 & 30.24 & 0.85 \\
$\infty$ & 56.73 & 0.00 & 56.73 & 0.46 \\
\hline \multicolumn{5}{c}{$\left.C: \min O b j_{2}\right)$} \\
\hline$\delta$ & $O b j_{1}+O b j_{2}$ & $O b j_{1}$ & $O b j_{2}$ & Time (s) \\
\hline 1 & 37.94 & 22.02 & 15.92 & 0.74 \\
2 & 37.03 & 21.10 & 15.92 & 1.63 \\
3 & 36.08 & 20.16 & 15.92 & 2.25 \\
$\infty$ & 37.30 & 21.38 & 15.92 & 1.97 \\
\hline \multicolumn{5}{c}{}
\end{tabular}

Table 3 shows the average computational results for the different objective functions and variations of $\delta$ over all problem instances. The table is divided into three blocks $A, B$, and $C$ to cover the different optimization goals. That is, we consider both objectives in $(A)$ and then split the objective function, as explained above, into its two composing parts, namely $O b j_{1}(B)$ and $O b j_{2}$ $(C)$. The three inner columns show the average objective function values assuming that one or the other objective is observed while the values are calculated also for the others. In the last column, the computational times in seconds are depicted. Given the computational results, the following implications can be derived.

- The separate minimization of $\mathrm{Obj}_{2}(C)$ is 
independent of parameter $\delta$. More precisely, the optimization of $O b j_{2}$ sets $\delta=1$ as can be seen from the other results where $O b j_{2}$ takes the same optimal values even when only $O b j_{1}(B)$ is minimized for the same $\delta$. However, we see that fixing $\delta$ leads to the highest overall objective values.

- The separate minimization of $O b j_{1}(C)$ shows that reallocations can be completely avoided for $\delta \geq 3$. This is due to the fact that the given instances, storage yard capacities, and the maximum closeness distance facilitate enough flexibility to find respective allocation plans. These allocation plans, however, do not take $\mathrm{Obj}_{2}$ into account leading to higher overall objective values $\left(O b j_{1}+O b j_{2}\right)$. To obtain overall optimal values, container reallocations are necessary for all of the scenarios as seen in $(A)$.

- The number of reallocations $\left(O b j_{1}\right)$ increases for lower values of $\delta$, as depicted in Figure 3. This can be expected as the number of reachable storage yards within the maximum allowed closeness set by $\delta$ decreases, making more reallocations necessary due to space limitations, especially because inbound and outbound groups need to be placed to yard areas close to the quayside. For the extreme case of $\delta=1$, where only two storage yards are available per terminal, the number of reallocations is the highest.

- Trade-off between $O b j_{1}$ and $O b j_{2}$ and contribution of objectives: The optimal values for $\mathrm{Obj}_{2}$ have the opposite tendency as they are decreasing for lower $\delta$ values compared to $O b j_{1}$, except of $(C)$. The reason is that more reallocations are necessary for achieving shorter distances between quayside and yard areas. The trade-off between the objectives is clearly observed in the cases for $\delta=3$ and $\delta=\infty$. When minimizing both objectives $(A)$, the resulting sum stays the same for both cases, because $O b j_{2}$ increases by the same amount as $O b j_{1}$ decreases. While for $\delta=1$ the optimal values of $O b j_{2}$ are the lowest and for $O b j_{1}$ the highest, respectively, $\mathrm{Obj}_{2}$ still contributes most to the overall result, as shown in Figure 4.

With respect to computational times, we see that the optimization of $\mathrm{Obj}_{2}(\mathrm{C})$ provides the highest performance, while the case of $\delta=2$ requires the longest average computational times as shown in Figure 5. By analyzing the computational times for separated objective functions, we see that the reason lies

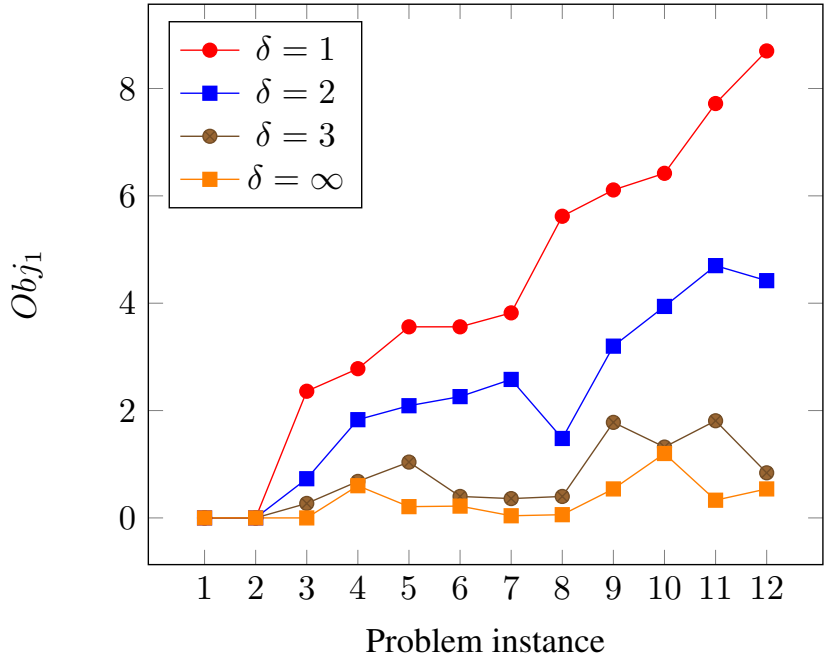

Figure 3. Impact of different values of $\delta$ on $O b j_{1}(A)$

in the minimization of $O b j_{1}$, requiring a high effort for the case of $\delta=2$, whereas the other cases take much less time to be solved to optimality. This situation is different for the simultaneous minimization of both objectives for which the largest instance takes more than 220 seconds to be solved and up to nearly twice as much for the case of $\delta=2$.

\section{Practical implications}

As discussed, the cases of $\delta=\infty$ and $\delta=3$ include the possibility of inter-terminal movements during unloading and loading processes, and thus can be considered impracticable in practice. Comparing the average results of $\delta=2$ and $\delta=1$ indicates an increase of merely $3.17 \%$ for the more restricted case of $\delta=1$. For a terminal operator, this slight increase could be considered acceptable as the minimization of vessels' handling times is one of the major competitive factors. Under the assumption that these handling times during the unloading and loading processes directly benefit from shorter travel distances, and therefore enable a higher overall terminal throughput, this implies that the terminal operator should choose the storage policy which limits this distance the most $(\delta=1)$.

Given the trade-off between both objectives, it has been shown that $O b j_{1}$ increases for more congested situations compared to the other instances. If more vessels and container groups need to be handled within the same time period, the limited storage capacity of the yards leads to the necessity of more reallocations in order to ensure that incoming and outgoing groups can be stored within the maximum distance of 


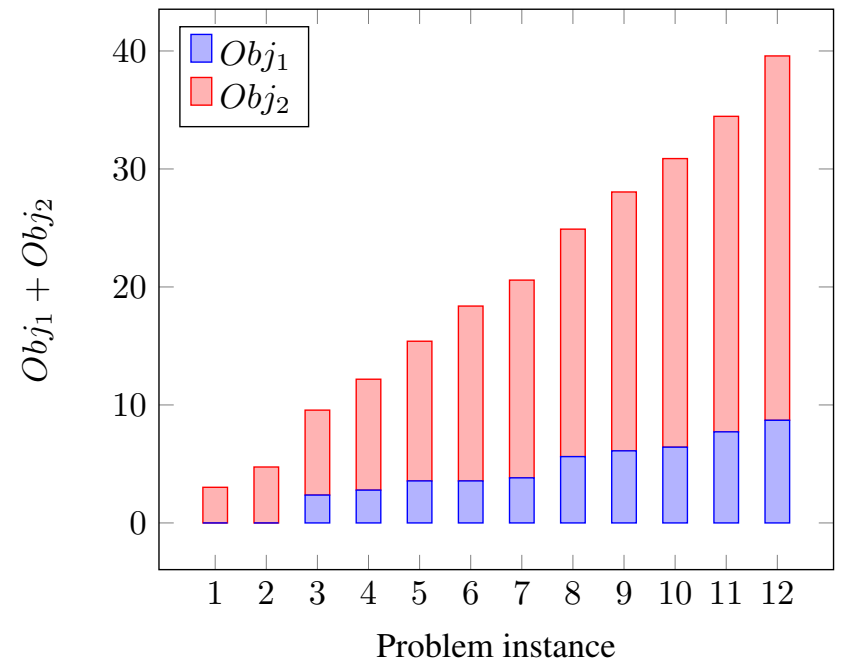

Figure 4. Impact of objectives for $\delta=1(A)$

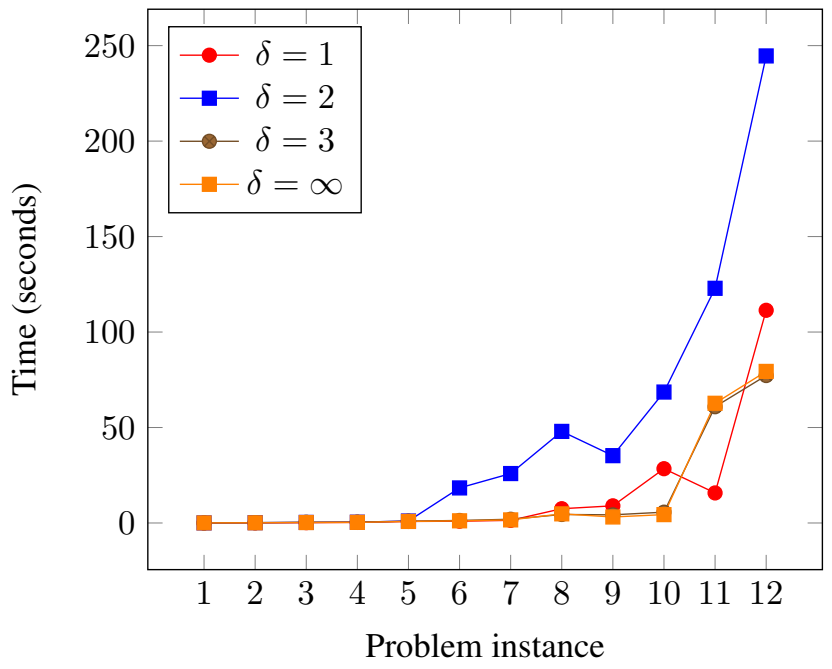

Figure 5. Average computational times $(A, B, C)$ their scheduled vessel. Therefore, adjustments of the vessel schedule might be beneficial and could be agreed with carriers using collaborative schemes. While a minimization of $O b j_{1}$, including the implicit minimization of $\mathrm{Obj}_{2}$ (with $\delta=1$ ), could be achieved in short computational times, in a quite congested situation the storage policy of $\delta=1$ might not be feasible as the capacity of the closest storage yards might be exceeded. An additional issue might be that the simultaneous minimization of both objectives might prove to not be solvable in reasonable time for large real-world problem instances, as indicated by the increasing computational times for experiment $(A)$.

Therefore, the sensitivity analysis has shown that terminal operators should prioritize the minimization of the second objective as it contributes most to the overall objective function value while taking the shortest time to be solved to optimality. These are the costs resulting from the storage processes during unloading and loading operations of a vessel based on the distance between berthing vessel and yard areas. Furthermore, as stated above, it can be assumed that this minimization also reduces the handling times which is an essential goal in the optimization of container terminal operations. Especially in congested situations, the results underline the importance of decision support for utilizing space in the yard while minimizing reallocations and travel distances for serving vessels more efficiently. For ports that suffer from peak demands, increasing vessel sizes, and limited space, such as the Port of Hamburg, simple allocation policies can have already an essential impact on the overall situation in the terminal and in the port environment.

\section{Conclusions and future research}

Approaches considering the operations of multiple container terminals are still rare, even though many operators have to manage multiple terminals of a port in reality. Especially the high interdependency between terminal and yard allocations requires decision support and policies in order to better utilize available resources and space. The scarcity of the latter is a growing problem in ports, in particular when inter-terminal transports occur as they further contribute to the traffic situation and congestion in and around ports. In container transshipment terminals, the storage of containers for inbound and outbound flows needs to be planned in a way that, on the one hand, the distance between quayside and yardside is kept short to ensure low vessel handling times, and, on the other hand, the distance of reallocations between yards is reduced while taking into account resource and storage capacities.

To address those issues, the TYAP, integrating the planning of vessels to terminals and the allocation of container groups to storage yards, has been studied for a multi-terminal transshipment hub in this work. By incorporating closeness limitations into the TYAP, the quay and yard container groups traveled distances have been studied under different closeness policies. This includes computational experiments in CPLEX to evaluate the impact of different parameter settings and components of the objective function on the optimal result and computational times. We analyzed the computational results from a practical point of view. While the minimization of traveled distances between quayside and yardside contributed the major part of the 
overall objective value, its computational complexity required the shortest time to be solved to optimality. A benefit that results from the minimization of this objective is that shorter travel distances for unloading and loading operations also reduce the required handling times, leading to a higher possible terminal throughput. This leads to the practical implication, that a terminal operator should prioritize the minimization of these travel distances upon berthing of a vessel. All of the twelve problem instances could be solved within reasonable computational time, leading to the conclusion that the optimization model can be applied to support the tactical decision making in the optimization of container terminal operations in a multi-terminal port. A deficit of this model, however, is the rather macroscopic point of view, performing the allocation to terminal and yards on the tactical level. Thus, in order to verify feasibility on the operational level, these results can serve as an input for other models that allocate the vessels to actual berthing positions and the containers to yard blocks within a terminal.

For future research, we aim to extend the problem formulation to consider more details of the berth allocation problem and container group movements, such as with respect to the vertical and horizontal transport resources (e.g., quay cranes and internal vehicles, respectively). Furthermore, by incorporating handling times into the model, the advantage of having shorter travel distances upon berthing, and their trade-off with the increased number of necessary reallocation movements, could be analyzed in more detail. Finally, a computational study of the mathematical model performance under different scenario sizes as well as the development of advanced decision support approaches for solving it will be a topic of future research.

\section{References}

[1] D.-H. Lee, J. G. Jin, and J. H. Chen, "Terminal and yard allocation problem for a container transshipment hub with multiple terminals," Transportation Research Part E: Logistics and Transportation Review, vol. 48, no. 2, pp. 516-528, 2012.

[2] K. Tierney, S. Voß, and R. Stahlbock, "A mathematical model of inter-terminal transportation," European Journal of Operational Research, vol. 235, no. 2, pp. 448-460, 2014.

[3] E. Lalla-Ruiz, J. de Armas, C. Expósito-Izquierdo, B. Melián-Batista, and J. M. Moreno-Vega, "A multi-stage approach aimed at optimizing the transshipment of containers in a maritime container terminal," in International Conference on Computer Aided Systems Theory, pp. 255-262, 2015.

[4] L. Heilig and S. Voß, "Inter-terminal transportation: an annotated bibliography and research agenda," Flexible
Services and Manufacturing Journal, vol. 29, no. 1, pp. 35-63, 2017.

[5] L. Heilig, E. Lalla-Ruiz, and S. Voß, "Multi-objective inter-terminal truck routing," Transportation Research Part E: Logistics and Transportation Review, vol. 106, pp. 178-202, 2017.

[6] L. Heilig, E. Lalla-Ruiz, and S. Voß, "port-IO: an integrative mobile cloud platform for real-time inter-terminal truck routing optimization," Flexible Services and Manufacturing Journal, vol. 29, no. 3-4, pp. 504-534, 2017.

[7] D. Steenken, S. Voß, and R. Stahlbock, "Container terminal operation and operations research - a classification and literature review," OR Spectrum, vol. 26, no. 1, pp. 3-49, 2004.

[8] X. Schepler, S. Balev, S. Michel, and É. Sanlaville, "Global planning in a multi-terminal and multi-modal maritime container port," Transportation Research Part E: Logistics and Transportation Review, vol. 100, pp. 38-62, 2017.

[9] M. Hendriks, D. Armbruster, M. Laumanns, E. Lefeber, and J. Udding, "Strategic allocation of cyclically calling vessels for multi-terminal container operators," Flexible Services and Manufacturing Journal, vol. 24, no. 3, pp. 248-273, 2012.

[10] N. Safaei, M. Bazzazi, and P. Assadi, "An integrated storage space and berth allocation problem in a container terminal," International Journal of Mathematics in Operational Research, vol. 2, no. 6, pp. 674-693, 2010.

[11] M. A. Salido, M. Rodriguez-Molins, and F. Barber, "Integrated intelligent techniques for remarshaling and berthing in maritime terminals," Advanced Engineering Informatics, vol. 25, no. 3, pp. 435-451, 2011.

[12] M. Hendriks, E. Lefeber, and J. Udding, "Simultaneous berth allocation and yard planning at tactical level," $O R$ Spectrum, vol. 35, no. 2, pp. 441-456, 2013.

[13] L. Zhen, E. P. Chew, and L. H. Lee, "An integrated model for berth template and yard template planning in transshipment hubs," Transportation Science, vol. 45, no. 4, pp. 483-504, 2011.

[14] D.-H. Lee, J. G. Jin, and J. H. Chen, "Schedule template design and storage allocation for cyclically visiting feeders in container transshipment hubs," Transportation Research Record, vol. 2273, no. 1, pp. 87-95, 2012.

[15] D.-H. Lee and J. G. Jin, "Feeder vessel management at container transshipment terminals," Transportation Research Part E: Logistics and Transportation Review, vol. 49, no. 1, pp. 201-216, 2013.

[16] J. G. Jin, D.-H. Lee, and H. Hu, "Tactical berth and yard template design at container transshipment terminals: A column generation based approach," Transportation Research Part E: Logistics and Transportation Review, vol. 73, pp. 168-184, 2015.

[17] Q. Zeng, Y. Feng, and Z. Chen, "Optimizing berth allocation and storage space in direct transshipment operations at container terminals," Maritime Economics \& Logistics, vol. 19, no. 3, pp. 474-503, 2017. 